\title{
AN OPTIMAL STRATEGY FOR THE CONTROL OF A TRAIN
}

\author{
PHIL HOWLETT ${ }^{1}$
}

(Received 2 September 1988; revised 10 April 1989)

\begin{abstract}
A train travels from one station to the next along a level track. The journey must be completed within a given time and it is desirable to minimise the energy required to drive the train. It has been shown with an appropriate formulation of the problem that an optimal strategy exists and that this strategy must satisfy a Pontryagin type criterion. In this paper the Pontryagin principle will be used to find the nature of the optimal strategy and this information will then be used to determine the precise optimal strategy.
\end{abstract}

\section{Introduction}

In 1977-78 Milroy [6] considered the problem of driving a train from one station to the next along a level track within a given allowable time in such a way that energy consumption is minimised. He formulated the problem as follows: Minimise the energy consumption

$$
I(u, v)=\int_{0}^{T} u_{+}(t) v(t) d t
$$

subject to the differential equation

$$
v^{\prime}(t)=u(t)-r[v(t)]
$$

with boundary conditions $v(0)=v(T)=0$ and subject to the equality constraint

$$
\int_{0}^{T} v(t) d t=L
$$

${ }^{1}$ School of Mathematics and computer Studies, South Australian Institute of Technology, The Levels Campus, South Australia.

(C) Copyright Australian Mathematical Society 1990, Serial-fee code 0334-2700/90 
and the inequality constraint $|u(t)| \leq 1$. In this formulation $T$ is the given time allowed for the journel and $L$ is the distance between the two stations, $u(t)$ is the acceleration applied to the train, $v(t)$ is the velocity and $r[v(t)]$ is the frictional resistance. It is assumed that a maximum applied acceleration is specified and that only positive acceleration consumes energy. Thus the cost functional contains the term

$$
u_{+}(t)=[u(t)+|u(t)|] / 2
$$

which is the positive part of $u(t)$. By applying the Pontryagin maximum principle Milroy obtained a basic velocity profile which he has suggested as an optimal strategy. This conjecture has been supported by more recent work of Tyler [7] and Kautsky et al [4] and by subsequent practical tests. However because he did not specify vector spaces for the various functions involved it was not possible for Milroy to justify his solution. In two previous papers Howlett [2] and [3] it was shown that when the problem is formulated in an appropriate function space an optimal strategy exists and the strategy does indeed satisfy a Pontryagin type criterion. The books by Yosida [8] and Luenberger [5] are suggested as useful references for the underlying functional analysis in these two papers and the methods of Craven [1] form a basis for the derivation of necessary conditions on an optimal strategy. In this paper the Pontryagin principle will be used to find the nature of the optimal strategy and this information will then be used to determine the precise optimal strategy. The problem will then be reformulated using only control strategies of optimal type and the simplified problem will be solved to show that each possible strategy is determined by a single real number parameter. A precise optimal strategy can now be found. In his original paper Milroy did not obtain a complete determination of the solution. Finally it should be noted that the cost functional used in this paper is more general than the cost functional used in the original formulation and consequently the optimal strategy is more complex.

\section{Formulation of the train control problem}

Let $\mathscr{B}$ denote the set of all real valued Borel measurable functions on the interval $[0, T]$. We consider two basic subsets of $\mathscr{B}$ and the associated Banach spaces. $\mathscr{U}$ is the subset of all essentially bounded functions. If we define a norm on this subset by the formula

$$
\|u\|_{\infty}=\underset{t \in[0, T]}{\operatorname{ess} . \sup }|u(t)|
$$


then $U=\left(\mathscr{U},\|\cdot\|_{\infty}\right)=L^{\infty}([0, T])$ is a Banach space. $\mathscr{V}$ is the subset of all essentially Lipschitz functions and for these functions a suitable norm is given by $\|v\|=\|v\|_{\infty}+\left\|v^{\prime}\right\|_{\infty}$. In this case $V=(\mathscr{V},\|\cdot\|)=C^{0,1}([0, T])$ is also a Banach space. It is convenient to introduce the subset

$$
\mathscr{V}_{0}=\{v \mid v \in \mathscr{V} \text { with } v(0)=v(T)=0\}
$$

and the subspace $V_{0}=\left(\mathscr{V}_{0},\|\cdot\|\right)$ which is again a Banach space. We will define a real valued cost functional $J$ on $\mathscr{U} \times \mathscr{V}$ by the formula

$$
J(u, v)=\int_{0}^{T} p[u(t)] q[v(t)] d t
$$

where $p: \mathbf{R} \rightarrow \mathbf{R}$ and $q: \mathbf{R} \rightarrow \mathbf{R}$ are functions with the following properties. The function $p$ is convex and piecewise linear and such that $p(u)=0$ when $u \leq 0$ and $p(u)>0$ when $u>0$. More precisely we will assume that there exist points $0=u_{1}<u_{2}<\cdots<u_{n}=1$ such that

$$
p(u)= \begin{cases}0 & (u<0) \\ a_{j}\left(u-u_{j}\right)+b_{j} & \left(u_{j} \leq u<u_{j+1}\right) \\ a_{n}(u-1)+b_{n} & (u \geq 1)\end{cases}
$$

where $0<a_{1}<a_{2}<\cdots<a_{n}, b_{1}=0$ and $b_{j+1}=a_{j}\left(u_{j+1}-u_{j}\right)+b_{j}$ for $j=1,2, \ldots, n-1$. The function $q$ is continuously differentiable with $q(0)=0$ and $q(v)$ strictly increasing when $v>0$. We will need some additional assumptions on $p$ and $q$ and these will be introduced in the next section. For the moment we observe that we wish to minimise $J(u, v)$ over all $u \in \mathscr{U}$ and $v \in \mathscr{V}_{0}$ subject to certain constraints. The first has the form

$$
\int_{0}^{T} v(t) d t=L
$$

and the second has the form

$$
v^{\prime}(t)=u(t)-r[v(t)]
$$

where the resistance function $r: \mathbf{R} \rightarrow \mathbf{R}$ is a function with $r(0)=0, r(v)$ strictly increasing for $v \geq 0$ and with $r(v) \downarrow r_{0}>0$ when $v \downarrow 0$. We will also assume that there is a continuously differentiable function $\bar{r}: \mathbf{R} \rightarrow \mathbf{R}$ with $\bar{r}(v)=r(v)$ when $v>0$. There are also two inequality constraints which must be observed. Firstly we need $|u(t)| \leq 1$ and secondly $v(t) \geq 0$. It is now possible to specify the problem precisely. Minimise the cost functional

$$
J(u, v)=\int_{0}^{T} p[u(t)] q[v(t)] d t
$$


over all $(u, v) \in \mathscr{U} \times \mathscr{Y}_{0}$ subject to the equality constraints $v^{\prime}(t)=u(t)-r[v(t)]$ and $\int_{0}^{T} v(t) d t=L$ and the inequality constraints $|u(t)| \leq 1$ and $v(t) \geq 0$.

\section{The nature of the optimal strategy}

We mentioned earlier that additional assumptions on $p$ and $q$ would be introduced. To this purpose we define functions $p_{j}: \mathbf{R} \rightarrow \mathbf{R}$ for each $j=$ $1,2, \ldots, n$ by setting $p_{j}(v)=a_{j}\left[r(v)-u_{j}\right]+b_{j}$ and if we use the notation $\left(p_{j} q\right)(v)$ to denote the product $p_{j}(v) q(v)$ then we will assume that $\left(p_{1} q\right)(v)$ and $\left(p_{n} q\right)(v)$ are strictly convex. It is now easy to establish that $\left(p_{j} q\right)(v)$ is strictly convex for each $j=1,2, \ldots, n$. With these additional assumptions we can now show that the adjoint differential equation

$$
\xi^{\prime}(t)-r^{\prime}\left[v_{0}(t)\right] \xi(t)=\tau p\left[u_{0}(t)\right] q^{\prime}\left[v_{0}(t)\right]+\sigma-w^{\prime}(t)
$$

and the Hamiltonian function

$$
h(u, t)=\tau p(u) q\left[v_{0}(t)\right]+\sigma v_{0}(t)+\xi(t)\left\{r\left[v_{0}(t)\right]-u\right\}
$$

can be used to determine the nature of the optimal journey. In this context $\tau \in \mathbf{R}$ and $\sigma \in \mathbf{R}$ are adjoint variables and $w: \mathbf{R} \rightarrow \mathbf{R}$ and $\xi: \mathbf{R} \rightarrow \mathbf{R}$ are functions used for the representation of adjoint variables by appropriate integrals. The function $v_{0}(t)$ is the optimal velocity of the train and the function $u_{0}(t)$ is the optimal applied acceleration.

From the previous paper [3] we know that the optimal acceleration $u_{0}$ can be obtained by minimising $h(u, t)$ over all $u \in I=[-1,1]$. We must therefore obtain one of the following basic situations.

$\left(\mathrm{T}_{1}\right): \tau a_{j-1} q\left[v_{0}(t)\right]-\xi(t)<0$ and $\tau a_{j} q\left[v_{0}(t)\right]-\xi(t)>0$, in which case

$$
h\left(u_{j}, t\right)=\min _{u \in I} h(u, t) \text {. }
$$

$\left(\mathrm{T}_{2}\right): \tau a_{j} q\left[v_{0}(t)\right]-\xi(t)=0$, in which case

$$
h\left(u^{*}, t\right)=\min _{u \in I} h(u, t) \text {, }
$$

for all $u^{*} \varepsilon\left[u_{j}, u_{j+1}\right]$.

$\left(\mathrm{T}_{3}\right): \xi(t)=0$, in which case

$$
h\left(u^{*}, t\right)=\min _{u \in I} h(u, t),
$$

for all $u^{*} \varepsilon[-1,0]$.

$\left(\mathrm{T}_{4}\right): \xi(t)<0$, in which case

$$
h(-1, t)=\min _{u \in I} h(u, t) .
$$

We will now state several results which allow us to develop the overall structure of the optimal journey. For convenience we will state them as a sequence of lemmas with only brief notes about the method of proof. 
LeMma 3.1. If $v_{0}(t)>0$ for all $t \in\left(t_{1}, t_{2}\right)$ then $w(t)$ is constant in this interval.

Proof. From the Fritz-John conditions (FJ) in [3] we know that $\nu^{*}\left(v_{0}\right)=0$, i.e. $\int_{[0, T]} v_{0}(t) w(d t)=0$ where $w(t)$ is increasing and where the integral is a Radon integral (see Howlett [3] and Yosida [8]). This condition derives from the requirement $v_{0}(t) \geq 0$. Now it follows that

$$
\begin{aligned}
0 & =\int_{[0, T]} v_{0}(t) w(d t) \\
& \geq \int_{\left[t_{1}+\delta, t_{2}-\delta\right]} v_{0}(t) w(d t) \\
& \geq \varepsilon\left[w\left(t_{2}-\delta\right)-w\left(t_{1}+\delta\right)\right]
\end{aligned}
$$

(some $\varepsilon>0$ ). Since $w(t)$ is increasing it must be constant.

LEMMA 3.2. If $\tau a_{j-1} q\left[v_{0}(t)\right]-\xi(t)<0$ and $\tau a_{j} q\left[v_{0}(t)\right]-\xi(t)>0$ for all $t \in\left(t_{1}, t_{2}\right)$ and if $u_{0}\left(t_{1}\right)=u_{j}>r\left[v_{0}\left(t_{1}\right)\right]$ then $u_{0}(t)=u_{j}>r\left[v_{0}(t)\right]$ for all $t \in\left[t_{1}, t_{2}\right]$.

Proof. Suppose there is some value $t_{3} \in\left(t_{1}, t_{2}\right]$ with $u_{j}=r\left[v_{0}\left(t_{3}\right)\right]$. For each $t \in\left(t_{1}, t_{3}\right)$ we have

$$
t=t_{1}+\int_{v_{0}\left(t_{1}\right)}^{v_{0}(t)}\left\{1 /\left[u_{j}-r(v)\right]\right\} d v
$$

But from the mean value theorem we can find $c_{v}$ with $v<c_{v}<v_{0}\left(t_{3}\right)$ such that $r\left[v_{0}\left(t_{3}\right)\right]-r(v)=r^{\prime}\left(c_{v}\right)\left[v_{0}\left(t_{3}\right)-v\right]$ and hence

$$
t>t_{1}+\varepsilon \int_{v_{0}\left(t_{1}\right)}^{v_{0}(t)}\left\{1 /\left[v_{0}\left(t_{3}\right)-v\right\} d v\right.
$$

where $\varepsilon>0$ is a lower bound for $1 / r^{\prime}(v)$ on the interval [ $v_{0}\left(t_{1}\right), v_{0}\left(t_{3}\right)$ ]. Clearly the RHS approaches infinity as $t \uparrow t_{3}$. Thus $t_{3}$ cannot be finite.

LEMMA 3.3. If $\tau a_{j} q\left[v_{0}(t)\right]-\xi(t)=0$ for all $t \in\left(t_{1}, t_{2}\right)$ then $v_{0}(t)=v_{0}\left(t_{1}\right)$ and $u_{0}(t)=r\left[v_{0}\left(t_{1}\right)\right]$ for all such $t$. This situation can only arise if $u_{j} \leq r\left[v_{0}\left(t_{1}\right)\right] \leq$ $u_{j+1}$.

Proof. Since $\tau a_{j} q\left[v_{0}(t)\right]-\xi(t)=0$ it follows that

$$
\tau a_{j} q^{\prime}\left[v_{0}(t)\right] v_{0}^{\prime}(t)-\xi^{\prime}(t)=0 .
$$

Using the adjoint equation and Lemma 3.1 this condition becomes

$$
\tau\left(p_{j} q\right)^{\prime}\left[v_{0}(t)\right]+\sigma=0,
$$

and since $\left(p_{j} q\right)(v)$ is strictly convex there is at most one value (say $V$ ) such that $\tau\left(p_{j} q\right)^{\prime}(V)+\sigma=0$. Thus $v_{0}(t)=V$ for all $t \in\left(t_{1}, t_{2}\right)$. 
LEMMA 3.4. If $\tau a_{j-1} q\left[v_{0}(t)\right]-\xi(t)<0$ and $\tau a_{j} q\left[v_{0}(t)\right]-\xi(t)>0$ for all $t \in$ $\left(t_{1}, t_{2}\right)$ and if $u_{0}(t)=u_{j}>r\left[v_{0}\left(t_{1}\right)\right]$ then it is not possible to have $\tau a_{j} q\left[v_{0}(t)\right]-$ $\xi(t)=0$ for all $t \in\left(t_{2}, t_{3}\right)$.

Proof. Suppose the contrary. From Lemma 3.2 we have $u_{j}>r\left[v_{0}\left(t_{2}\right)\right]$ and from Lemma 3.3 we have $u_{j} \leq r\left[v_{0}\left(t_{2}\right)\right]$ which is a contradiction.

LEMMA 3.5. If $\tau a_{j-1} q\left[v_{0}(t)\right]-\xi(t)<0$ and $\tau a_{j} q\left[v_{0}(t)\right]-\xi(t)>0$ for all $t \in\left(t_{1}, t_{2}\right)$ and if $u_{0}(t)=u_{j}>r\left[v_{0}\left(t_{1}\right)\right]$ on this interval then it is not possible to have $\tau a_{j} q\left[v_{0}(t)\right]-\xi(t)<0$ and $\tau a_{j+1} q\left[v_{0}(t)\right]-\xi(t)>0$ for all $t \in\left(t_{2}, t_{3}\right)$.

Proof. Suppose the contrary. From Lemma 3.2 it is clear that $u_{j}>r\left[v_{0}\left(t_{2}\right)\right]$ and since $u_{j+1}>u_{j}>r\left[v_{0}\left(t_{2}\right)\right]$ it is also clear that $u_{j+1}>r\left[v_{0}\left(t_{3}\right)\right]$. Thus $v_{0}(t)$ increases on $\left(t_{1}, t_{3}\right)$. We also know that $\tau a_{j} q\left[v_{0}(t)\right]-\xi(t)$ is positive on $\left(t_{1}, t_{2}\right)$ and negative on $\left(t_{2}, t_{3}\right)$. Thus for all $t \in\left(t_{2}, t_{3}\right)$ we have

$$
\begin{aligned}
\tau a_{j} q^{\prime}\left[v_{0}(t)\right] v_{0}^{\prime}(t)-\xi^{\prime}(t) & <(-1)\left\{\tau\left(p_{j} q\right)^{\prime}\left[v_{0}(t)\right]+\sigma\right\} \\
& \leq(-1)\left\{\tau\left(p_{j} q\right)^{\prime}\left[v_{0}\left(t_{2}\right)\right]+\sigma\right\} \\
& =\tau a_{j} q^{\prime}\left[v_{0}\left(t_{2}\right)\right] v_{0}^{\prime}\left(t_{2}\right)-\xi^{\prime}\left(t_{2}\right),
\end{aligned}
$$

since $\tau a_{j} q\left[v_{0}\left(t_{2}\right)\right]-\xi\left(t_{2}\right)=0$. Now we know that $\tau a_{j} q\left[v_{0}(t)\right]-\xi(t)$ is decreasing at $t=t_{2}$ and hence

$$
\tau a_{j} q^{\prime}\left[v_{0}\left(t_{2}\right)\right] v_{0}^{\prime}\left(t_{2}\right)-\xi^{\prime}\left(t_{2}\right) \leq 0 .
$$

Thus we have shown that $\tau a_{j} q\left[v_{0}(t)\right]-\xi(t)$ is negative and decreasing throughout the interval $\left(t_{2}, t_{3}\right)$. Let us suppose that the optimal strategy changes at $t=t_{3}$. From Lemma 3.4 we can see that it is not possible to have $\tau a_{j+1} q\left[v_{0}(t)\right]-\xi(t)=0$ for all $t \in\left(t_{3}, t_{4}\right)$ and so the only possible change would require $\tau a_{j+1} q\left[v_{0}(t)\right]-\xi(t)<0$ on $\left(t_{3}, t_{4}\right)$. A continuation of this argument will show that such a journey can never terminate.

Although the above results do not constitute a complete determination of the overall structure of the optimal journey they do indicate the methods that must be used. For example Lemma 3.2 shows us that when condition (T1) is valid on a time interval $\left[t_{1}, t_{2}\right]$ and if the optimal acceleration $u_{0}(t)=u_{j}$ exceeds the frictional resistance $r\left[v_{0}(t)\right]$ at the beginning of the time interval then it exceeds the frictional resistance at the end of the time interval as well. Lemma 3.4 shows us that such a situation can only terminate (at $t=t_{2}$ ) with the condition $\tau a_{j-1} q\left[v_{0}\left(t_{2}\right)\right]-\xi\left(t_{2}\right)=0$. Thus on a subsequent time interval $\left(t_{2}, t_{3}\right)$ we must have either $\tau a_{j-1} q\left[v_{0}(t)\right]-\xi(t)=0$ or else we have both $\tau a_{j-1} q\left[v_{0}(t)\right]-\xi(t)<0$ and $\tau a_{j} q\left[v_{0}(t)\right]-\xi(t)>0$ throughout the interval. In the former case condition (T2) holds and we can apply Lemma 3.2 (with $j$ 
replaced by $j-1)$ to see that a constant acceleration $u_{0}(t)=r\left[v_{0}(t)\right]=r(V) \leq$ $u_{j}$ is applied throughout the interval. In the latter case condition (T1) again applies (but with $j$ replaced by $j-1$ and) with acceleration $u_{0}(t)=u_{j-1}$ throughout the interval. Lemma 3.5 shows that it is not possible for the level of acceleration to be given by $u_{0}(t)=u_{j}$ on a particular time interval and then to be given by $u_{0}(t)=u_{j+1}$ on an immediately subsequent time interval. In summary the level of ontimal acceleration must progressively decrease with the passage of time.

Thus the optimal journey must consist of an acceleration phase (during which time $u_{0}(t)=1 \rightarrow u_{0}(t)=u_{n-1} \rightarrow u_{0}(t)=u_{n-2} \rightarrow \cdots \rightarrow u_{0}(t)=$ $u_{n-k+1}$ ) followed by a speed holding phase (when $u_{0}(t)=r\left[v_{0}(t)\right]$ ) and a subsequent coasting phase (during which time $u_{0}(t)=u_{n-k} \rightarrow u_{0}(t)=u_{n-k-1} \rightarrow$ $\cdots \rightarrow u_{0}(t)=0$ and the velocity decreases "gradually"). Finally there is a braking phase (with $u_{0}(t)=-1$ ). It is possible to have an optimal journey in which certain stages are omitted.

\section{The complete solution}

We will consider an optimal journey with the acceleration phase defined by

$$
u_{0}(t)=u_{n-i} \quad \text { for } t \in\left(\sum_{r=0}^{i} \alpha_{r}, \sum_{r=0}^{i+1} \alpha_{r}\right)
$$

and each $i=0,1,2, \ldots, k-1$. We will define the velocities $0=V_{0}<V_{1}<$ $\cdots<V_{k}$ by the formulae

$$
V_{i}=v_{0}\left(\sum_{r=0}^{i} \alpha_{r}\right)
$$

for each $i=0,1,2, \ldots, k$. We will take $\alpha_{0}=0$ with $\alpha_{r} \geq 0$ for the remaining values of $r$. The speedholding phase will be given by

$$
u_{0}(t)=r\left(V_{k}\right) \text { for } t \in\left(\sum_{r=0}^{k} \alpha_{r}, \sum_{r=0}^{k} \alpha_{r}+\beta\right)
$$

where $\beta \geq 0$. The coasting phase is specified by

$$
u_{0}(t)=u_{n-(k+j)} \quad \text { for } t \in\left(\sum_{r=0}^{k} \alpha_{r}+\beta+\sum_{s=0}^{j} \gamma_{s}, \sum_{r=0}^{k} \alpha_{r}+\beta+\sum_{s=0}^{j+1} \gamma_{s}\right)
$$

and each $j=0,1,2, \ldots, n-k-1$. We define the velocities $V_{k}>V_{k+1}>\cdots>$ $V_{n} \geq 0$ by the formulae

$$
V_{k+j}=v_{0}\left(\sum_{r=0}^{k} \alpha_{r}+\beta+\sum_{s=0}^{j} \gamma_{s}\right)
$$


for each $j=0,1,2, \ldots, n-k$. We will assume $\gamma_{0}=0$ with $\gamma_{s} \geq 0$ for the remaining values of $s$. Finally the braking phase is defined by

$$
u_{0}(t)=-1 \quad \text { for } t \in\left(\sum_{r=0}^{k} \alpha_{r}+\beta+\sum_{s=0}^{n-k} \gamma_{s}, \sum_{r=0}^{k} \alpha_{r}+\beta+\sum_{s=0}^{n-k} \gamma_{s}+\delta\right)
$$

where the final velocity $V_{n+1}=0$ is defined by

$$
V_{n+1}=v_{0}\left(\sum_{r=0}^{k} \alpha_{r}+\beta+\sum_{s=0}^{n-k} \gamma_{s}+\delta\right) \text {. }
$$

The actual functions used in the synthesis of $v_{0}(t)$ are defined in the following way. For each $i=0,1,2, \ldots, k-1$ we define $t_{i}:\left[0, V_{i}^{*}\right) \rightarrow[0, \infty)$ by the formula

$$
t_{i}(v)=\int_{0}^{v}\left\{1 /\left[u_{n-i}-r(w)\right]\right\} d w,
$$

where $V_{i}^{*}$ is the unique point with $r\left(V_{i}^{*}\right)=u_{n-i}$. We can choose a constant $r_{i}$ such that $u_{n-i}-r(w) \leq r_{i}\left(V_{i}^{*}-w\right)$ for all $w \in\left[0, V_{i}^{*}\right)$. Thus $t_{i}(v) \geq$ $\left(1 / r_{i}\right) \ln \left[1-\left(v / V_{i}^{*}\right)\right]$ and hence $t_{i}(v) \uparrow \infty$ as $v \uparrow V_{i}^{*}$. Now if we define $\left[v_{i}^{*}\right]:[0, \infty) \rightarrow\left[0, V_{i}^{*}\right)$ as the inverse function of $t_{i}$ then it is easy to see that $\left[v_{i}^{*}\right]^{\prime}(t)=u_{n-i}-r\left\{\left[v_{i}^{*}\right](t)\right\}$. Furthermore it is clear that $\left[v_{i}^{*}\right](t) \uparrow V_{i}^{*}$ as $t \uparrow \infty$. Since $0 \leq V_{i}<V_{i}^{*}$ we can define the acceleration phase of the optimal journey by the formulae

$$
v_{0}(t)=\left[v_{i}^{*}\right]\left(t-\sum_{r=0}^{i} \alpha_{r}+t_{i}\left[V_{i}\right]\right) \quad \text { for } t \in\left(\sum_{r=0}^{i} \alpha_{r}, \sum_{r=0}^{i+1} \alpha_{r}\right)
$$

and each $i=0,1,2, \ldots, k-1$. Incidentally it is clear that $V_{i+1}=\left[v_{i}^{*}\right]\left(\alpha_{i+1}+\right.$ $\left.t_{i}\left[V_{i}\right]\right)$. For the speedholding phase of the optimal journal it is clear that

$$
v_{0}(t)=V_{k} \quad \text { for } t \in\left(\sum_{r=0}^{k} \alpha_{r}, \quad \sum_{r=0}^{k} \alpha_{r}+\beta\right) .
$$

Now for each $j=0,1,2, \ldots, n-k-2$ we define $t_{k+j}:\left(V_{k+j}^{*}, V_{k}\right] \rightarrow[0, \infty]$ by the formula

$$
t_{k+j}(v)=\int_{v}^{V_{k}}\left\{1 /\left[r(w)-u_{n-k-j}\right]\right\} d w,
$$

where $V_{k+j}^{*}$ is the unique point with $r\left(V_{k+j}^{*}\right)=u_{n-k-j}$. (It is necessary to assume at this stage that $u_{2}>r_{0}$ i.e. the lowest non zero level of acceleration is assumed sufficient to overcome the initial resistance to motion.) We can choose a constant $r_{k}$ so that $r(w)-u_{n-k-j} \leq r_{k}\left(w-V_{k+j}^{*}\right)$ for all $w \in\left(V_{k+j}^{*}, V_{k}\right]$ and so

$$
t_{k+j}(v) \geq\left(1 / r_{k}\right) \ln \left[\left(V_{k}-V_{k+j}^{*}\right) /\left(v-V_{k+j}^{*}\right)\right]
$$


and hence $t_{k+j}(v) \uparrow \infty$ as $v \downarrow V_{k+j}^{*}$. Now we define $\left[v_{k+j}^{*}\right]:[0, \infty) \rightarrow\left(V_{k+j}^{*}, V_{k}\right]$ as the inverse function of $t_{k+j}$ and it is easy to see that

$$
\left[v_{k+j}^{*}\right]^{\prime}(t)=u_{n-k-j}-r\left\{\left[v_{k+j}^{*}\right](t)\right\} .
$$

Furthermore it is clear that $\left[v_{k+j}^{*}\right](t) \downarrow V_{k+j}^{*}$ as $t \uparrow \infty$. Since $V_{k} \geq V_{k+j}>V_{k+j}^{*}$ we can define all stages except the final stage for the coasting phase of the ontimal journey by the formulae

$$
\begin{gathered}
v_{0}(t)=\left[v_{k+j}^{*}\right]\left(t-\left[\sum_{r=0}^{k} \alpha_{r}+\beta+\sum_{s=0}^{j} \gamma_{s}\right]+t_{k+j}\left[V_{k+j}\right]\right) \\
\text { for } t \in\left(\sum_{r=0}^{k} \alpha_{r}+\beta+\sum_{s=0}^{j} \gamma_{s}, \quad \sum_{r=0}^{k} \alpha_{r}+\beta+\sum_{s=0}^{j+1} \gamma_{s}\right)
\end{gathered}
$$

for each $j=0,1,2, \ldots, n-k-2$. For the final stage of the coasting phase we begin by defining $t_{n-1}:\left[0, V_{k}\right] \rightarrow\left[0, t_{n-1}(0)\right]$ by the formula

$$
t_{n-1}(v)=\int_{v}^{V_{k}}\{1 / r(w)\} d w
$$

Since $r(w) \geq r_{0}>0$ it follows that $t_{n-1}(v) \leq\left(V_{k}-v\right) / r_{0}$. Now the inverse function $\left[v_{n-1}^{*}\right]:\left[0, t_{n-1}(0)\right] \rightarrow\left[0, V_{k}\right]$ satisfies the differential equation

$$
\left[v_{n-1}^{*}\right]^{\prime}(t)=(-1) r\left\{\left[v_{n-1}^{*}\right](t)\right\},
$$

and since $V_{k} \geq V_{n-1}>0$ we can define the final stage of the coasting phase by the formula

$$
\begin{gathered}
v_{0}(t)=\left[v_{n-1}^{*}\right]\left(t-\left[\sum_{r=0}^{k} \alpha_{r}+\beta+\sum_{s=0}^{n-k-1} \gamma_{s}\right]+t_{n-1}\left[V_{n-1}\right]\right) \\
\text { for } t \in\left(\sum_{r=0}^{k} \alpha_{r}+\beta+\sum_{s=0}^{n-k-1} \gamma_{s}, \sum_{r=0}^{k} \alpha_{r}+\beta+\sum_{s=0}^{n-k} \gamma_{s}\right) .
\end{gathered}
$$

Incidentally we can now see that for all $j=0,1,2, \ldots, n-k-1$ we have $V_{k+j+1}=\left[v_{k+j}^{*}\right]\left(\gamma_{j+1}+t_{k+j}\left[V_{k+j}\right]\right)$. For the braking phase of the optimal journey we can begin by defining $t_{n}:\left[0, V_{k}\right] \rightarrow\left[0, t_{n}(0)\right]$ by the formula

$$
t_{n}(v)=\int_{v}^{V_{k}}\{1 /[r(w)+1] d w .
$$

Since $r(w)+1 \geq r_{0}+1>0$ it follows that $t_{n}(v) \leq\left(V_{k}-v\right) /\left(r_{0}+1\right)$. Now the inverse function $\left[v_{n}^{*}\right]:\left[0, t_{n}(0)\right] \rightarrow\left[0, V_{k}\right]$ satisfies the differential equation

$$
\left[v_{n}^{*}\right]^{\prime}(t)=(-1)-r\left\{\left[v_{n}^{*}\right](t)\right\}
$$


and since $V_{k} \geq V_{n} \geq 0$ we can define the braking phase of the optimal journey by the formula

$$
\begin{gathered}
v_{0}(t)=\left[v_{n}^{*}\right]\left(t-\left[\sum_{r=0}^{k} \alpha_{r}+\beta+\sum_{s=0}^{n-k} \gamma_{s}\right]+t_{n}\left[V_{n}\right]\right) \\
\text { for } t \in\left(\sum_{r=0}^{k} \alpha_{r}+\beta+\sum_{s=0}^{n-k} \gamma_{s}, \sum_{r=0}^{k} \alpha_{r}+\beta+\sum_{s=0}^{n-k} \gamma_{s}+\delta\right),
\end{gathered}
$$

where $\delta$ is chosen so that $V_{n+1}=\left[v_{n}^{*}\right]\left(\delta+t_{n}\left[V_{n}\right]\right)=0$. Hence $\delta=t_{n}(0)-t_{n}\left(V_{n}\right)$ depends on $\alpha, \beta$ and $\gamma$.

Now that we can describe the basic format of the optimal journey it is possible to formulate the problem in a more amenable form. The cost of the optimal journal is given by

$$
\begin{aligned}
J_{0}(\alpha, \beta, \gamma)= & \sum_{i=0}^{k-1} \int_{0}^{\alpha_{i+1}} p\left(u_{n-i}\right) q\left\{\left[v_{i}^{*}\right]\left(\tau+t_{i}\left[V_{i}\right]\right)\right\} d \tau+p\left\{r\left(V_{k}\right)\right\} q\left(V_{k}\right) \beta \\
& +\sum_{j=0}^{n-k-1} \int_{0}^{\gamma_{j+1}} p\left(u_{n-k-j}\right) q\left\{\left[v_{k+j}^{*}\right]\left(\tau+t_{k+j}\left[V_{k+j}\right]\right)\right\} d \tau,
\end{aligned}
$$

and the distance travelled during the optimal journal is given by

$$
\begin{aligned}
d(\alpha, \beta, \gamma)= & \sum_{i=0}^{k-1} \int_{0}^{\alpha_{i+1}}\left[v_{i}^{*}\right]\left(\tau+t_{i}\left[V_{i}\right]\right) d \tau+V_{k} \beta \\
& \left.+\sum_{j=0}^{n-k-1} \int_{0}^{\gamma_{j+1}}\left[v_{k+j}^{*}\right]\left(\tau+t_{k+j}\left[V_{k+j}\right]\right)\right\} d \tau \\
& +\int_{0}^{t_{n}(0)-t_{n}\left(V_{n}\right)}\left[v_{n}^{*}\right]\left(\tau+t_{n}\left[V_{n}\right]\right) d \tau .
\end{aligned}
$$

Therefore we can now consider the original problem in the following form. Minimise $J_{0}(\alpha, \beta, \gamma)$ subject to the (equality) constraint $d(\alpha, \beta, \gamma)=L$ and the (inequality) constraints $\alpha \geq 0, \beta \geq 0, \gamma \geq 0$ and $\sum_{r=1}^{k} \alpha_{r}+\beta+\sum_{s=0}^{n-k} \gamma_{s}+$ $t_{n}(0)-t_{n}\left(V_{n}\right) \leq T$.

Thus we form a Langrangean function

$$
\begin{aligned}
\mathcal{F}_{0}(\alpha, \beta, \gamma ; \lambda, \mu, \nu, \eta, \xi)= & J_{0}(\alpha, \beta, \gamma)+\lambda[L-d(\alpha, \beta, \gamma)] \\
& \cdot\left[\sum_{r=1}^{k} \mu_{r} \alpha_{r}+\nu \beta+\sum_{s=1}^{n-k} \eta_{s} \gamma_{s}\right] \\
& +\xi\left[\sum_{r=1}^{k} \alpha_{r}+\beta+\sum_{s=1}^{n-k} \gamma_{s}+t_{n}(0)-t_{n}\left(V_{n}\right)-T\right] .
\end{aligned}
$$


The necessary conditions for optimality are now the Kuhn-Tucker equations viz.

$$
\partial \mathscr{I}_{0} / \partial \alpha_{r}=0, \quad \partial \mathscr{I}_{0} / \partial \beta=0, \quad \partial \mathscr{I}_{0} / \partial \gamma_{s}=0
$$

with $\lambda[L-d(\alpha, \beta, \gamma)]=0, \mu_{r} \alpha_{r}=0, \nu \beta=0, \eta_{s} \gamma_{s}=0$, and

$$
\xi\left[\sum_{r=1}^{k} \alpha_{r}+\beta+\sum_{s=1}^{n-k} \gamma_{s}+t_{n}(0)-t_{n}\left(V_{n}\right)-T\right]=0
$$

with $\mu_{r} \geq 0, \nu \geq 0, \eta_{s} \geq 0$ and $\xi \geq 0$.

In order to calculate the above derivatives efficiently we must first establish some convenient formulae. When $m<r+1$ we have

$$
\begin{aligned}
\frac{\partial V_{r+1}}{\partial \alpha_{m}} & \left.=\frac{\partial}{\partial \alpha_{m}}\left\{\left[v_{r}^{*}\right]\left(\alpha_{r+1}+t_{r}\left[V_{r}\right]\right)\right]\right\} \\
& =\left[v_{r}^{*}\right]^{\prime}\left(\alpha_{r+1}+t_{r}\left[V_{r}\right]\right) t_{r}^{\prime}\left(V_{r}\right) \frac{\partial V_{r}}{\partial \alpha_{m}} \\
& =\frac{u_{n-r}-r\left(V_{r+1}\right)}{u_{n-r}-r\left(V_{r}\right)} \frac{\partial V_{r}}{\partial \alpha_{m}},
\end{aligned}
$$

while we also have

$$
\begin{aligned}
\frac{\partial V_{m}}{\partial \alpha_{m}} & =\frac{\partial}{\partial \alpha_{m}}\left\{\left[v_{m-1}^{*}\right]\left(\alpha_{m}+t_{m-1}\left[V_{m-1}\right]\right)\right\} \\
& =\left[v_{m-1}^{*}\right]^{\prime}\left(\alpha_{m}+t_{m-1}\left[V_{m-1}\right]\right) \\
& =u_{n-m+1}-r\left(V_{m}\right) .
\end{aligned}
$$

Hence by induction we obtain the general formula

$$
\frac{\partial V_{i}}{\partial \alpha_{m}}=\prod_{r=m}^{i-1} \frac{u_{n-r+1}-r\left(V_{r}\right)}{u_{n-r}-r\left(V_{r}\right)}\left[u_{n-i+1}-r\left(V_{i}\right)\right]
$$

whenever $m<i$. We can extend this result by noting that

$$
\begin{aligned}
\frac{\partial V_{k+s+1}}{\partial \alpha_{m}} & =\frac{\partial}{\partial \alpha_{m}}\left\{\left[v_{k+s}^{*}\right]\left(\gamma_{s+1}+t_{k+s}\left[V_{k+s}\right]\right)\right\} \\
& =\left[v_{k+s}^{*}\right]^{\prime}\left(\gamma_{s+1}+t_{k+s}\left[V_{k+s}\right]\right) t_{k+s}^{\prime}\left(V_{k+s}\right) \frac{\partial V_{k+s}}{\partial \alpha_{m}} \\
& =\frac{u_{n-k-s}-r\left(V_{k+s+1}\right)}{u_{n-k-s}-r\left(V_{k+s}\right)} \frac{\partial V_{k+s}}{\partial \alpha_{m}}
\end{aligned}
$$


from which it follows by induction that

$$
\frac{\partial V_{k+j}}{\partial \alpha_{m}}=\prod_{s=0}^{j-1} \frac{u_{n-k-s}-r\left(V_{k+s+1}\right)}{u_{n-k-s}-r\left(V_{k+s}\right)} \frac{\partial V_{k}}{\partial \alpha_{m}}
$$

for each $j=1,2, \ldots, n-k$. Now the previous result can be used to give

$$
\begin{aligned}
\frac{\partial V_{k+j}}{\partial \alpha_{m}} & =\prod_{s=0}^{j-1} \frac{u_{n-k-s}-r\left(V_{k+s+1}\right)}{u_{n-k-s}-r\left(V_{k+s}\right)} \prod_{r=m}^{k-1} \frac{u_{n-r+1}-r\left(V_{r}\right)}{u_{n-r}-r\left(V_{r}\right)}\left[u_{n-k+1}-r\left(V_{k}\right)\right] \\
& =\prod_{r=m}^{k+j-1} \frac{u_{n-r+1}-r\left(V_{r}\right)}{u_{n-r}-r\left(V_{r}\right)}\left[u_{n-k-j+1}-r\left(V_{k+j}\right)\right] .
\end{aligned}
$$

In similar fashion when $l<s+1$ we can see that

$$
\begin{aligned}
\frac{\partial V_{k+s+1}}{\partial \gamma_{l}} & =\frac{\partial}{\partial \gamma_{l}}\left\{\left[v_{k+s}^{*}\right]\left(\gamma_{s+1}+t_{k+s}\left[V_{k+s}\right]\right)\right\} \\
& =\left[v_{k+s}^{*}\right]^{\prime}\left(\gamma_{s+1}+t_{k+s}\left[V_{k+s}\right]\right) t_{k+s}^{\prime}\left(V_{k+s}\right) \frac{\partial V_{k+s}}{\partial \gamma_{l}} \\
& =\frac{u_{n-k-s}-r\left(V_{k+s+1}\right)}{u_{n-k-s}-r\left(V_{k+s}\right)} \frac{\partial V_{k+s}}{\partial \gamma_{l}} .
\end{aligned}
$$

Since it is also clear that

$$
\begin{aligned}
\frac{\partial V_{k+l}}{\partial \gamma_{l}} & =\frac{\partial}{\partial \gamma_{l}}\left\{\left[v_{k+l-1}^{*}\right]\left(\gamma_{l}+t_{k+l-1}\left[V_{k+l-1}\right]\right)\right\} \\
& =\left[v_{k+l-1}^{*}\right]^{\prime}\left(\gamma_{l}+t_{k+l-1}\left[V_{k+l-1}\right]\right) \\
& =u_{n-k-l+1}-r\left(V_{k+l}\right),
\end{aligned}
$$

we can again use induction to obtain the general formula

$$
\frac{\partial V_{k+j}}{\partial \gamma_{l}}=\prod_{s=l}^{j-1} \frac{u_{n-k-s+1}-r\left(V_{k+s}\right)}{u_{n-k-s}-r\left(V_{k+s}\right)}\left[u_{n-k-j+1}-r\left(V_{k+j}\right)\right]
$$

whenever $l<j$. By applying these formulae in the calculation of the appropriate partial derivatives we obtain a suitable form for the Kuhn-Tucker equations. 
In fact we have

$$
\begin{aligned}
\partial \mathscr{J}_{0} / \partial \alpha_{m}= & p\left(u_{n-m+1}\right) q\left\{\left[v_{m-1}^{*}\right]\left(\alpha_{m}+t_{m-1}\left[V_{m-1}\right]\right)\right\} \\
& +\sum_{i=m}^{k-1} \int_{0}^{\alpha_{i+1}} p\left(u_{n-i}\right) q^{\prime}\left\{\left[v_{i}^{*}\right]\left(\tau+t_{i}\left[V_{i}\right]\right)\right\} \\
& \times\left[v_{i}^{*}\right]^{\prime}\left(\tau+t_{i}\left[V_{i}\right]\right) t_{i}^{\prime}\left(V_{i}\right) \frac{\partial V_{i}}{\partial \alpha_{m}} d \tau \\
& +\left\{p^{\prime}\left[r\left(V_{k}\right)\right] r^{\prime}\left(V_{k}\right) q\left(V_{k}\right)+p\left[r\left(V_{k}\right)\right] q^{\prime}\left(V_{k}\right)\right\} \frac{\partial V_{k}}{\partial \alpha_{m}} \beta \\
& +\sum_{j=0}^{n-k-1} \int_{0}^{\gamma_{j+1}} p\left(u_{n-k-j}\right) q^{\prime}\left\{\left[v_{k+j}^{*}\right]\left(\tau+t_{k+j}\left[V_{k+j}\right]\right)\right\} \\
& -\lambda\left\{\left[v_{m-1}^{*}\right]\left(\alpha_{m}+t_{m-1}\left[V_{m-1}\right]\right)\right. \\
& +\sum_{i=m}^{k-1} \int_{0}^{\alpha_{i+1}}\left[v_{i}^{*}\right]^{\prime}\left(\tau+t_{i}\left[V_{i}\right]\right) t_{i}^{\prime}\left(V_{i}\right) \frac{\partial V_{i}}{\partial \alpha_{m}} d \tau+\frac{\partial V_{k}}{\partial \alpha_{m}} \beta \\
& \left.+\sum_{j=0}^{n-k-1} \int_{0}^{\gamma_{j+1}}\left[v_{k+j}^{*}\right]\left(\tau+t_{k+j}\right]\right) t_{k+j}^{\prime}\left(V_{k+j}\right) \frac{\partial V_{k+j}}{\partial \alpha_{m}} d \tau \\
& -\left[v_{n}^{*}\right]\left(t_{n}\left[V_{n}\right]\right) t_{n}^{\prime}\left(V_{n}\right) \frac{\partial V_{n}}{\partial \alpha_{m}}-\mu_{m}+\xi\left[1-t_{n}^{\prime}\left(V_{n}\right) \frac{\partial V_{n}}{\partial \alpha_{m}}\right]=0
\end{aligned}
$$

This equation can now be rewritten in the simplified form $\left(E_{1}\right)$ below

$$
\begin{aligned}
p\left(u_{n-m+1}\right) q\left(V_{m}\right)+\sum_{i=m}^{n-1} p\left(u_{n-i}\right)\left[q\left(V_{i+1}\right)-q\left(V_{i}\right)\right] \prod_{r=m}^{i} \frac{u_{n-r+1}-r\left(V_{r}\right)}{u_{n-r}-r\left(V_{r}\right)} \\
\quad+\left(p_{n-k} q\right)^{\prime}\left(V_{k}\right) \prod_{r=m}^{k-1} \frac{u_{n-r+1}-r\left(V_{r}\right)}{u_{n-r}-r\left(V_{r}\right)}\left[u_{n-k+1}-r\left(V_{k}\right)\right] \beta \\
\quad-\lambda\left\{V_{m}+\sum_{i=m}^{n}\left[V_{i+1}-V_{i}\right] \prod_{r=m}^{i} \frac{u_{n-r+1}-r\left(V_{r}\right)}{u_{n-r}-r\left(V_{r}\right)}\right. \\
\left.+\prod_{r=m}^{k-1} \frac{u_{n-r+1}-r\left(V_{r}\right)}{u_{n-r}-r\left(V_{r}\right)}\left[u_{n-k+1}-r\left(V_{k}\right)\right] \beta\right\} \\
\quad-\mu_{m}+\xi\left[1-\prod_{r=m}^{n} \frac{u_{n-r+1}-r\left(V_{r}\right)}{u_{n-r}-r\left(V_{r}\right)}\right]=0,
\end{aligned}
$$

for each $m$ with $1 \leq m \leq k$. A much less complicated calculation now gives

$$
\frac{\partial \mathscr{J}_{0}}{\partial \beta}=p\left\{r\left(V_{k}\right)\right\} q\left(V_{k}\right)-\lambda V_{k}-\nu+\xi=0,
$$


from which we obtain the equation $\left(E_{2}\right)$ in the form

$$
\left(p_{n-k} q\right)\left(V_{k}\right)-\lambda V_{k}-\nu+\xi=0 .
$$

Finally we calculate

$$
\begin{aligned}
\frac{\partial \mathscr{I}_{0}}{\partial \gamma_{l}}= & p\left(u_{n-k-l+1}\right) q\left\{\left[v_{k+l-1}^{*}\right]\left(\gamma_{l}+t_{k+l-1}\left[V_{k+l-1}\right]\right)\right\} \\
& +\sum_{j=l}^{n-k-1} \int_{0}^{\gamma_{j+1}} p\left(u_{n-k-j}\right) q^{\prime}\left\{\left[v_{k+j}^{*}\right]\left(\tau+t_{k+j}\left[V_{k+j}\right]\right)\right. \\
& \times\left[v_{k+j}^{*}\right]^{\prime}\left(\tau+t_{k+j}\left[V_{k+j}\right]\right) t_{k+j}^{\prime}\left(V_{k+j}\right) \frac{\partial V_{k+j}}{\partial \gamma_{l}} d \tau \\
& -\lambda\left\{\left[v_{k+l-1}^{*}\right]\left(\gamma_{l}+t_{k+l-1}\left[V_{k+l-1}\right]\right)\right. \\
& +\sum_{j=l}^{n-k-1} \int_{0}^{\gamma_{j+1}}\left[v_{k+j}^{*}\right]^{\prime}\left(\tau+t_{k+j}\left[V_{k+j}\right]\right) t_{k+j}^{\prime}\left(V_{k+j}\right) \frac{\partial V_{k+j}}{\partial \gamma_{l}} d \tau \\
& -\eta_{l}+\xi\left[1-t_{n}^{\prime}\left(V_{n}\right) \frac{\partial V_{n}}{\partial \gamma_{l}}\right]=0,
\end{aligned}
$$

and note that this equation can be rewritten in the form $\left(E_{3}\right)$ given below:

$$
\begin{aligned}
p\left(u_{n-k-l+1}\right) q\left(V_{k+l}\right)+\sum_{j=l}^{n-k-1} p\left(u_{n-k-j}\right)\left[q\left(V_{k+j+1}\right)-q\left(V_{k+j}\right)\right] \\
\times \prod_{s=l}^{j} \frac{u_{n-k-s+1}-r\left(V_{k+s}\right)}{u_{n-k-s}-r\left(V_{k+s}\right)} \\
-\lambda\left\{V_{k+l}+\sum_{j=l}^{n-k}\left[V_{k+j+1}-V_{k+j}\right] \prod_{s=l}^{j} \frac{u_{n-k-s+1}-r\left(V_{k+s}\right)}{u_{n-k-s}-r\left(V_{k+s}\right)}\right\} \\
-\eta_{l}+\xi\left[1-\prod_{s=l}^{n-k} \frac{u_{n-k-s+1}-r\left(V_{k+s}\right)}{u_{n-k-s}-r\left(V_{k+s}\right)}\right]=0,
\end{aligned}
$$

for each $l$ with $1 \leq l \leq n-k$. For convenience in the above formulae we have used the notation $u_{0}=-1$ wherever necessary. In order to solve the equations given by the Kuhn-Tucker conditions we assume to begin with that $\alpha, \beta$ and $\gamma$ are positive. Thus we must have $\mu=0, \nu=0$ and $\eta=0$. If we take $\left(E_{3}\right)$ with $l=n-k$ it follows that $\xi=\lambda V_{n}$.

Thus $\xi=0$ only if $\lambda=0$ or $V_{n}=0$ and from $\left(E_{2}\right)$ it is clear that $\lambda=0$ is not possible. If we put $l=n-k-1$ in $\left(E_{3}\right)$ we obtain

$$
\left(p_{1} q\right)\left(V_{n-1}\right)-\lambda\left[V_{n-1}-V_{n}\right]=0 \text {, }
$$


and if we subsequently use an inductive argument on $\left(E_{3}\right)$, then it follows that

$$
\left(p_{j} q\right)\left(V_{n-j}\right)-\lambda\left[V_{n-j}-V_{n}\right]=0,
$$

for each $j=1,2, \ldots, n-k-1$. From equation $\left(E_{2}\right)$ we get

$$
\left(p_{n-k} q\right)\left(V_{k}\right)-\lambda\left[V_{k}-V_{n}\right]=0 .
$$

If these results are used in conjunction with equation $\left(E_{1}\right)$ in the case $m=k$ then it can be seen that

$$
\left(p_{n-k} q\right)^{\prime}\left(V_{k}\right)=\lambda \text {. }
$$

With $m=k-1$ in equation $\left(E_{1}\right)$ we find that

$$
\left(p_{n-k+1} q\right)\left(V_{k-1}\right)-\lambda\left[V_{k-1}-V_{n}\right]=0,
$$

and once again an inductive argument, applied this time to equation $\left(E_{1}\right)$, can be used to deduce that

$$
\left(p_{n-k+i} q\right)\left(V_{k-i}\right)-\lambda\left[V_{k-i}-V_{n}\right]=0,
$$

for each $i=1,2, \ldots, k-1$. These results can be written collectively in the form

$$
\frac{\left(p_{j-1} q\right)\left(V_{n-j+1}\right)-\left(p_{j} q\right)\left(V_{n-j}\right)}{V_{n-j+1}-V_{n-j}}=\lambda
$$

for each $j=1,2, \ldots, n-1$. In this form (4) compares in nature with (3). If we have either $\alpha_{m}=0$ (for some $m$ ) or $\gamma_{l}=0$ (for some $l$ ) then the corresponding stages are simply omitted. The fundamental nature of the results is however the same and the same formulae apply. The notation must be adjusted to allow for a re-numbering of the appropriate stages but if we imagine that the whole problem is simply reworked with the null stages omitted it is easy to see that the same analysis will again apply. Similar comments can be made when $\beta=0$ and the speedholding phase is omitted. This time however the results are changed to the extent that equation (3) will be deleted.

In the case of a journey that contains a speedholding phase it can now be seen that the maximum velocity $V^{*}$ achieved (during the speedholding phase) on the journey is sufficient to determine the parameter $\lambda$ and the velocities $V_{1}, V_{2}, \ldots, V_{n}$. For each such type of optimal journey (i.e. for each selected configuration of null stages) and a given maximum velocity $V^{*}$ the time allowed for the speedholding phase can be adjusted to achieve the appropriate value of $d\left(V^{*}, \beta\right)$ viz. $d\left(V^{*}, \beta^{*}\right)=L$. Of course this may not be possible for all types of optimal journey and in cases where it is possible there may be a violation of the time constraint. When both the distance and time constraints can be satisfied the optimal type journey is feasible and the cost 
[16]

An optimal strategy for the control of a train

469

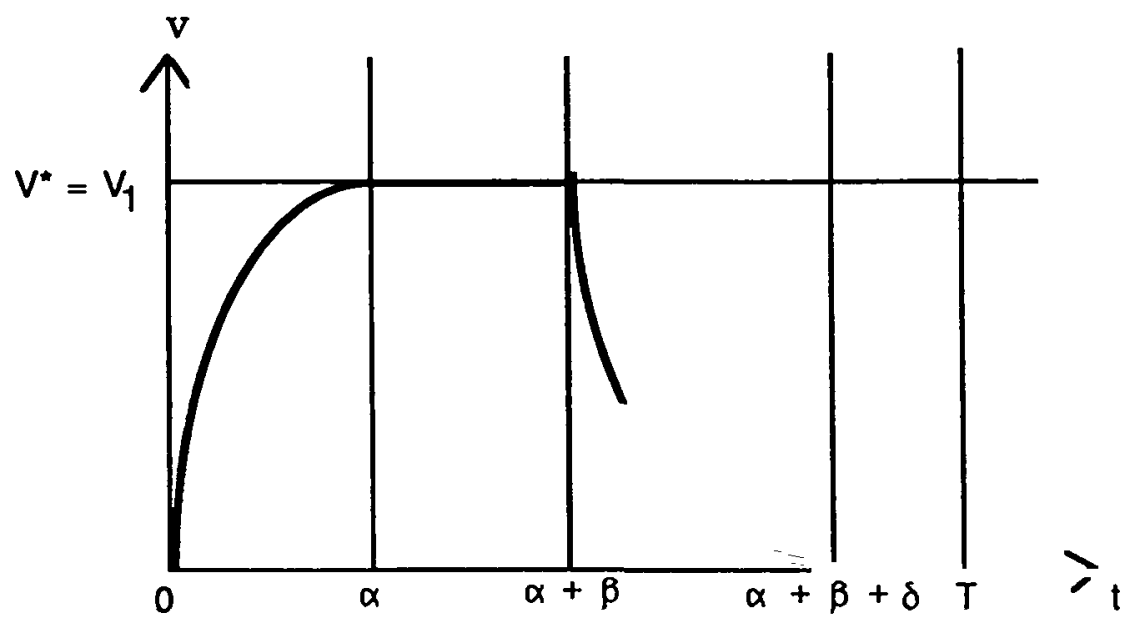

FIGURE 1. A simple trajectory involving maximum acceleration, with speedholding and maximum braking. The length of the speedholding phase is not specified.

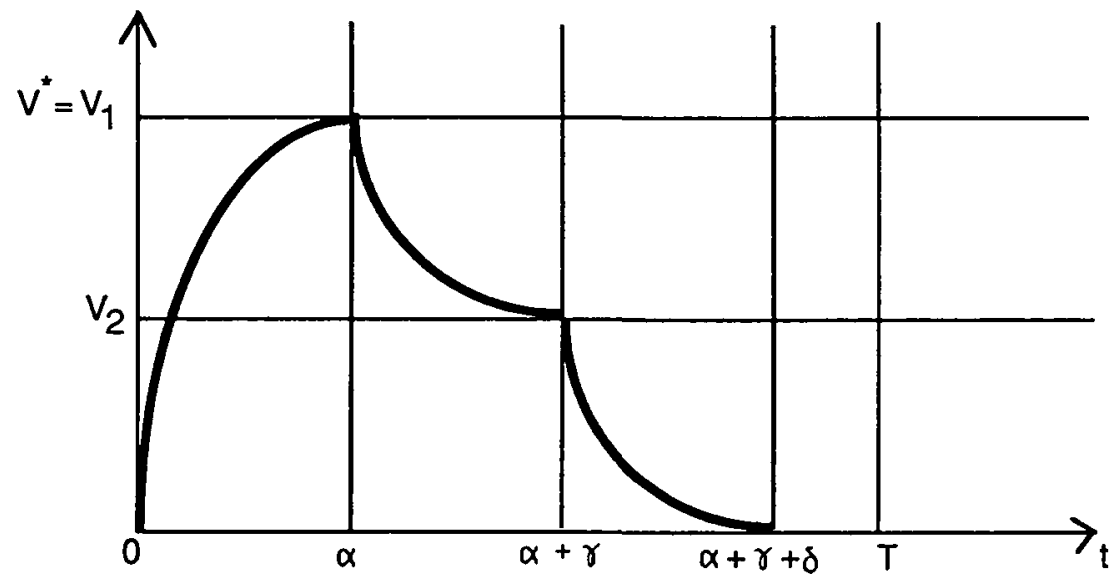

FIGURE 2. A simple trajectory involving maximum acceleration, zero acceleration (coasting) and maximum braking but with no speedholding. The velocity at which braking begins is not specified.

can be calculated. The first diagram shows an optimal type velocity profile and shows how the distance travelled, represented by the area under the curve, can be adjusted by varying the time allowed for the speedholding phase. In the case of a journey that contains no speedholding phase the value of the parameter $\lambda$ is no longer specified by the value $V^{*}$ of the maximum velocity. For each such optimal journey the value of the parameter $\lambda$ determines the velocities $V_{1}, V_{2}, \ldots, V_{n}$ from $V^{*}$ and consequently determines

https://doi.org/10.1017/S0334270000006780 Published online by Cambridge University Press 
the distance travelled. Thus we must now choose $\lambda$ so that $d\left(V^{*}, \lambda\right)=L$. Alternatively we can see that determination of any one value $V_{i}$ (other than the value $V_{k}=V^{*}$ ) will determine $\lambda$ and hence determine all other values $V_{j}$. Provided that the time constraint is satisfied, this journey will be feasible and can then be costed. The second diagram shows an optimal type velocity profile with no speed holding phase and shows that selection of the velocity at which braking begins will detcrinine the cômpiete journey.

We can now calculate the cost of each feasible type of optimal journey and so determine the minimum cost journey.

\section{Conclusions}

This paper provides a clear answer to the type of strategy that must be adopted to achieve a minimum cost journey. The optimal strategy involves successive levels of constant applied acceleration with each subsequent level less than the preceding one and with the allowable levels restricted to the points at which the slope of the piecewise linear function $p(u)$ changes. If the maximum velocity of the optimal journey is given, then this paper shows that a single real number parameter determines the complete journey. The appropriate value of this parameter can be determined by satisfying the distance requirement and the journey will then be regarded as feasible if the time constraint is not violated. No specific method is suggested for the parameter determination and further work could be done to develop an efficient numerical procedure. In the case of the more general problem where the function $p(u)$ is not piecewise linear it seems reasonable to assume that an approximate optimal journey could be obtained by using a piecewise linear approximation to $p(u)$.

\section{Acknowledgements}

I would like to thank Ian Milroy for communicating the problem and Isobel Keegan for typing the original manuscript.

\section{References}

[1] B. D. Craven, Mathematical programming and control theory (Chapman and Hall, 1978),

[2] Phil Howlett, "Existence of an optimal strategy for the control of a train", South Australian Institute of Technology, School of Mathematics Report (1987). 
[3] Phil Howlett, "Necessary conditions on an optimal strategy for the control of a train", South Australian Institute of Technology, School of Mathematics Report (1987).

[4] J. Kautsky, A. M. Long, and I. P. Milroy, "Energy Conservation of Rail Vehicles", Report to the Institution of Engineers, Australia (1983).

[5] D. G. Luenberger, Optimization by vector space methods (Wiley, 1969).

[6] 1. P. Milroy, “Aspects of automatic train control”, Loughborough University Ph.D. Thesis, 1980.

[7] K. Tyler, "Energy minimization of rail vehicles", South Australian Institute of Technology, Master of Applied Science Thesis, 1982.

[8] K. Yosida, Functional analysis (5th ed. Springer, 1978). 